\section{DLBCL: Konditionierung myeloablativ oder nicht?}

In der Third-Line-Therapie des diffusen großzelligen B-Zell-Lymphoms (DLBCL) ist die allogene Stammzelltransplantation eine Option. Der Nutzen wurde jedoch nie in großen Studien geklärt; nun gibt es eine retrospektive Analyse.

$E_{\text {und }}^{\text {the }}$ wa $80 \%$ aller Patienten mit DLBCL - und anderen aggressiven Lymphomen werden durch moderne Therapien geheilt. Von denen, die keine Remission erreichen oder ein Rezidiv entwickeln, können einige durch Hochdosis-Chemotherapie und autologe Stammzelltransplantation gerettet werden. Als Third-Line-Therapie wird an manchen Zentren auch eine allogene Stammzelltransplantation angeboten.

Aufgrund relativ geringer Fallzahlen und der großen histologischen Heterogenität von DLBCL ist die Rolle der allogenen Stammzelltransplantation ungeklärt. Zwar lässt sich bei 40-50\% der Patienten mit myeloablativer Konditionierung (MAC) ein Langzeitüberleben erreichen, aber die transplantationsbedingte Mortalität ist mit 30-40\% hoch. Womöglich könnten eine Konditionierung mit reduzierter Intensität (RIC) oder eine nicht myeloablative Konditio- nierung (NMAC) zu besseren Ergebnissen führen. Ulrike Bacher und Kollegen untersuchten retrospektiv den Krankheitsverlauf von 396 Empfängern einer allogenen Stammzelltransplantation nach verschiedenen Konditionierungsregimes (MAC 165, RIC 143, NMAC 88).

Akute und chronische Graft-versusHost-Reaktionen (GvHD) waren in allen Gruppen ähnlich häufig. Die nicht rezidivbedingte Mortalität nach fünf Jahren war nach MAC höher als nach RIC und NMAC (56 vs. 47 vs. $36 \%$; $\mathrm{p}=$ 0,007), die Rezidiv- bzw. Progressionsraten nach fünf Jahren waren dafür nach MAC niedriger (26 vs. 38 vs. $40 \%$; $\mathrm{p}=0,031)$. Progressionsfreies (15-25\%) und Gesamtüberleben nach fünf Jahren (18-26\%) unterschieden sich nicht signifikant.

In multivariaten Analysen ergab sich, dass NMAC-Regimes und eine noch nicht so lang zurückliegende Transplan- tation mit einem niedrigeren nicht rezidivbedingten Mortalitätsrisiko einhergingen; ein niedriger Karnofsky-Performance-Index, ein bereits aufgetretenes, therapieresistentes Rezidiv und ein nicht verwandter Donor waren dagegen mit einer höheren nicht rezidivbedingten Mortalität verbunden.

Fazit: Eine sanftere Konditionierung kann bei ausgewählten DLBCL-Patienten zu einem langen progressionsfreien Überleben führen; das Rezidivrisiko ist aber höher als bei aggressiverer Konditionierung. Es scheint, als würden jüngere DLBCL-Patienten und Patienten mit geringer Komorbidität von einer stärkeren Konditionierung profitieren; ältere Patienten oder solche mit schweren Komorbiditäten könnten dagegen für sanftere Regimes selektiert werden. Die Daten müssen aber vorsichtig interpretiert werden, da es sich lediglich um eine retrospektive Registeranalyse handelt.

Christina Berndt

Bacher $U$ et al. Conditioning regimens for allotransplants for diffuse large B-cell lymphoma: Myeloablative or reduced intensity? Blood. 2012;120(20):4256-62.

\section{Lymphom: Bestrahlung verbessert Kontrolle}

Patienten mit diffusem großzelligem B-Zell-Lymphom (DLBCL) im Frühstadi-
um erhalten routinemäßig eine Radiotherapie zur Konsolidierung. Warum
sollte diese Strategie nicht auch bei weiter fortgeschrittenen Tumoren helfen?

D ie kontroverse Debatte, ob die beim DLBCL im Frühstadium angewendete Konsolidierungsradiotherapie auch Patienten mit fortgeschrittenem DLBCL helfen kann, untermauern Kollegen aus North Carolina, USA, nun mit Daten. Sie analysierten retrospektiv die Ergebnisse von 79 Patienten mit DLBCL im Stadium III-IV, die zwischen 1991 bis 2009 an der Duke University behandelt worden waren und vollständig auf die Chemotherapie angesprochen hatten. Sie verglichen Patienten mit und ohne Radiotherapie nach der Chemotherapie.

$65 \%$ der Patienten hatten R-CHOP bekommen (Cyclophosphamid, Doxorubicin, Vincristin und Prednison plus Ritu- ximab). Weitere $22 \%$ der Patienten erhielten lediglich CHOP und $13 \%$ eine andere Chemotherapie. Der Erfolg der Chemotherapie wurde mittels Bildgebung festgestellt, bei den meisten Patienten (73\%) mit einer Kombination aus Positronenemissionstomografie und Computertomografie (PET/CT), bei 14\% mit Gallium-CT und bei $13 \%$ mit CT. 38 Patienten (48\%) erhielten eine InvolvedField-Radiotherapie zur Konsolidierung (im Median 25 Gray).

Bei diesen Patienten war die betroffene Region häufiger kontrollierbar (92 vs. $69 \% ; \mathrm{p}=0,028)$ und das Gesamtüberleben besser ( 85 vs. $65 \%$; $p=0,014$ ) als bei Patienten ohne Radiotherapie. Das Ge- samtüberleben unterschied sich jedoch nicht signifkant ( 85 vs. $78 \%$; $p=0,15$ ). In einer multivariaten Analyse war der Nichterhalt einer Radiotherapie prädiktiv für ein erhöhtes Risiko von lokoregionalem Therapieversagen (Hazard Ratio [HR] 8,01; $\mathrm{p}=0,014)$ sowie für ein kürzeres krankheitsfreies Überleben (HR $4,3 ; \mathrm{p}=0,014)$.

Fazit: Patienten mit DLBCL im Stadium III-IV, die vollständig auf Chemotherapie ansprechen, profitieren im Hinblick auf krankheitsfreies Überleben und die betroffene Region von einer niedrigdosierten Konsolidierungsradiotherapie. Sie nützte auch Patienten mit kleineren Tumoren, die im Durchschnitt nur fünf Zentimeter maßen.

Christina Berndt

Dorth JA et al. Impact of consolidation radiation therapy in stage III-IV diffuse large B-cell lymphoma with negative post-chemotherapy radiologic imaging. Int J Radiat Oncol Biol Phys. 2012;84(3):762-7. 